\title{
CrimRxiv
}

\section{Identifying a spatial scale for the analysis of residential burglary: An empirical framework based on point pattern analysis}

Mohammed A. Alazawi, Shiguo Jiang, Steven F. Messner

Published on: Mar 01, 2022

DOI: $10.21428 / \mathrm{cb} 6 a b 371.691 \mathrm{e} 1 \mathrm{~d} 02$

License: Creative Commons Attribution 4.0 International License (CC-BY 4.0). 
